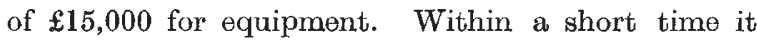
will therefore be well equipped with standard instruments, glassware, etc., for botanical, zoological, physiological, biochemical and hydrographical investigations and for educational purposes, whereas visitors must be preparod to bring special instruments and microscopes as well as special or rare chemicals and personal laboratory equipment.

All laboratories have running cold and hot freshMater as well as circulating sea-water. Gas, compressed air, etc., are provided by small transportable units. 220.V. alternating current is available. When furnishing the laboratories stress has been laid on great elasticity, permitting a rapid change from one type of laboratory to another.

The scientific staff is small. The Station is mainly a visitors' research laboratory, open all the year round for research workers from all countries. The visitors will get a laboratory or a table, material, instruments, chemicals and all the service that the Station can afford, all free of charge on condition that they publish their results in one of the publications of the
University, unless dispensation has been given. The material collected belongs to the Station, and all types and duplicates of rare species have to be handed over to the Station after description. Furthermore, visitors must be prepared to pay for particularly expensive collecting trips and costly chemicals. Board and lodging will be available at a low price.

Application for a table should be sent to the director, if possible one month in advance (for the months June-August not later than May 1). Exceptionally the Station can send material for investigation in other laboratories on the same conditions as those mentioned above.

The Station will give courses in botany, zoology and marine biology for students from the Universities in Bergen and Oslo, and also for students from the other Scandinavian countries. The last-named courses are part of a joint Scandinavian programme for education and research recently started. The Scandinavian countries also exchange scholarship holders.

\title{
BIOLOGY OF YEAST
}

$\mathrm{D}$ URING the British Association meoting at Dublin, Sections B (Chemistry) and $K$ (Botany) held a joint session on September 9, at which "The Biology of Yeast" was discussed. Prof. D. G. Catcheside read the first paper, which was on "The Geneties of Yeast". He said that although regular transmission of genetic traits to daughter cells shows that an orderly process of chromosome division exists in yeasts, the details of the mitotic process, and even the identification of nuclei and other cellular organelles, have not yet been satisfactorily settled. Yeast has a simple lifocyclo with an alternation of haploid (sexual) and diploid (asexual) generations. The haploid cells belong to one or other of two opposite mating types, determined by Mendelizing genes, and possessing some of the attributes of different sexes. When haploid cells of opposite mating type are mixed, they become associated in pairs and fuse to form zygotes, within which the two haploid nuclei fuse. Under conditions favouring growth the zygotes germinate to produce the diploid phase which can later be caused to sporulate by starvation. The mating types segregate characteristically in sporulation. The main differenco botween this process and the genetical cycle common to most higher plants and animals is that the segregation products of any one event of meiosis are kept as a group of four ascospores in the yeast ascus, and if these ascospores are isolated they reproduce vegetatively. This results in a substantial gain in genetical information, so that genetical analysis may be applied to discovering details in the life-history of yeast which have so far eluded the cytologist who studies these problems with a microscope.

Complexities may be introduced into inheritance patterns because, first, the mating types are able to mutate, though rarely, from one to the other, and, secondly, the haploids can undergo chromosome doubling by an unexplained process to produce diploids of either mating type. When the latter take part in sexual reproduction, triploids or tetra- ploids are formed, and these show complicated genetic segregations at sporulation, and this has in the past led to difficulty in interpretation of results. When sporulation occurs in triploids or tetraploids, mating type segregates in a trisomic or tetrasomic manner, and in either case the frequency of each type of ascus depends upon the position of the gene in the chromosome with respect to the centromere. The extreme cases are when crossing-over between the locus and the centromere of its chromosome is either zero or limiting; any particular case is likely to be intermediate between these two limits. This obviously leads to situations which cannot be recognized and distinguished without careful genetic analysis, lack of which has led to confusion in yeast genetics.

The number of chromosomes in yeast can be determined by two distinct genetical methods. The first method depends on the determination of how many linkage groups there are, while the second depends on the formation and analysis of an aneuploid zygote which is trisomic for one chromosome and disomic for the rest, so that, on sporulation, characteristic trisomic and disomic ratios may be obtained for genes located on the respective chromosomes; a disomic ratio for one factor occurring with a trisomic ratio for a second factor shows that the two factors must bo in different chromosomes. At least eight linkage groups have been recognized so far by these mothods, putting a lower limit of eight to the chromosome number. The recent discovery and use of linear asci should give valuable information on reduction division and the frequency with which reduction occurs at the first and second division of meiosis. By a detailed discussion of one group of inherited characters (adenine dependence, red colour) some of the techniques and results of genetic analysis were illustrated.

Prof. E. J. Conway then read a paper on "Cell Structure and Function in Yeast". Having outlined the principal structural features of the yeast cell, he 
pointed out that the cell membrane and its functions had been subjected to intensive investigation, and that this was not surprising as one of the most interesting features of the internal environment of this organism is its very high osmotic pressure. It is clear that there must be some mechanism which allows ions to reach the interior of the cell but prevents them from coming out again, and at least three explanations of this mechanism have been suggested : passage through pores in the membrane; solution in a lipid layer of the cell membrane; and active transport which involves temporary combination with a chemical entity at one side of the membrane and with consequent liberation at the other side. The 'cylindrical pore' theory has been investigated by finding the selective permeability of the membrane in terms of the relative sizes of the ions, due regard being had to the facts that hydration may alter the relative sizes of ions and that electrical forces depending on membrane potential difference and ion valency may affect rate of penetration of ions. It is generally true that larger ions pass through membranes only with difficulty, but no quantitative relations have been established. Danielli's theory of a double lipid layer with protein monolayers has been investigated by comparing rates of penetration of substances with their lipid solubility, and, although there is a general proportionality, no exact relation holds.

The active transport theory was then discussed by Prof. Conway with reference to studies made in his laboratory on the mechanism of acid production by yeast, on the nature of the acid produced, on the effect of acid production on the environment of the cell, and on the transport of cations across the yeast cell wall. During the fermentation of unbuffered glucose solution, yeast produces acid by two processes : liberation of succinic acid (in the absence of salts) and exchange of hydrogen ions formed internally for potassium ions (when potassium is present in the medium). The latter process, which is accompanied by a quantitative increase in bicarbonate ions in the cell, results in an accumulation of potassium ions in the yeast and an outside $p \mathrm{H}$ falling as low as 1.4. The experimental results are best explained on the basis of a redox system-the socalled redox pump mechanism. This hypothesis requires the existence of a mobile redox system within the cell membrane which, having been reduced, say by metabolic hydrogen, would complex with a cation at the internal boundary of the membrane; this complex would then orient itself to the external boundary, where on transferring its electrons to an acceptor system it would release the cation to the medium. It is possible to replace the internal potas. sium of the yeast cell by sodium or ammonium and so make the interesting 'sodium yeasts' and 'ammonium yeasts'; these interchanges are readily reversed. Although we have gained some insight into how the cell membrane functions, a description of the complete pattern of organization within the yeast cell must await further knowledge of the functioning of its subcellular components.

The third paper, on "The Biochemistry of Alcoholic Fermentation", was read by Dr. R. Davies. He referred to the fact that it was just one hundred years since Louis Pasteur published his first paper on alcoholic fermentation, in which fermentation was defined as life without air. Since then it has been found that fermentation depends on a series of internal oxidations and reductions in which energy is released in a form in which the organism can use it for synthesis of cell material. The energy is stored principally in high-energy phosphates of which a typical member is adenosine triphosphate. Because of the high energy of the terminal phosphate bond, this can act as a link between the energy-liberating metabolic reactions and the energy-requiring synthetic reactions. The fermentation of sugar by yeasts involves four phases: first the passage of the sugar across the outer cell membrane into the cell ; second, the preparation of the sugar for entry into the energyyielding mechanism proper; third, degradation to ethanol and carbon dioxide; and fourth, synthesis of cell materials. Two molecules of adenosine triphosphate are used up in phase two (this is the energy required to set the process going); four are released in phase three, so that there is an overall gain of two molecules of adenosine triphosphate per molecule of glucose fermented ; these highenergy phosphates are then available for synthetic work in phase four. The series of reactions in phases two and three, which are known as the EmbdenMyerhof-Parnas glycolysis system, involve the chain glucose, glucose-6-phosphate, fructose-6-phosphate, fructose-1 : 6-diphosphate, phosphoglyceraldehyde (and dihydroxyacetone phosphate), $1: 3$-diphosphoglyceric acid, 3-phosphoglyceric acid, 2-phosphoglyceric acid, 2-phosphoenolpyruvic acid, pyruvic acid, acetaldehyde (and carbon dioxide), and finally ethyl alcohol. The interconversion of each pair of these intermediates requires its own enzyme and frequently a coenzyme. The action of these enzymes accounts for alcoholic fermentation of glucose or fructose, but for the fermentation of galactose at least three further enzymes are required, and these enzymes may correspond with three dominant genes shown by genetical investigations to be involved in galactose fermentation. In the passage of sugar into the cell (phase one) it has been found that the cell membranes exhibit highly specific permeabilities and that some ions pass into the cell only by active transport, which takes place while the cell is metabolizing. The uptake of amino-acids, and of certain $\beta$-galactosides, by Escherichia coli involves active transport but also appears to be mediated by substances having all the properties of enzymes which have been called permeases. For yeast, only the uptake of $\alpha$-methylglucoside has so far been shown to involve permease action. It may be that the formation of permeases is genetically controlled and it is possible that if rate of fermentation of a sugar is limited by permease activity, this may provide an explanation of cases in which an oligosaccharide ferments at a higher rate than its component hexoses.

The fourth paper, on "Yeast Biology in Relation to Fermentation Techniques", was read by Mr. R. B. Gilliland. He first mentioned the many industries interested either in yeast production or in the products of fermentation, and went on to say that studies of the genetics, biochemistry, cytology and physiology of yeast have contributed to the improve. ment of industrial techniques and the solution of industrial problems. As examples of this, the improvement of yeast strains, the production of strains with new properties, the control of the balance between flocculated and dispersed yeast, and the control of the balance between aerobic respiration and anaerobic fermentation, were discussed. Con- 
siderable improvements in the performance of yeasts have been made by strain selection and, more recently, by the production of new hybrid strains. Yeast appears to have an advantage over other organisms in that its haploid generation can have a free existence, and may be tested, so that a selection of the best haploids may be used for hybrid production. This apparent advantage is largely illusory, as any character except the very simplest may be governed by many genes, so that the ability of a diploid cannot be judged by the separate abilities of its haploid compononts. The best mothod of finding better strains may therefore be to screen large numbers of randomly produced hybrids. Several producers of baking yeast have already found artificially produced yeast hybrids which are superior to naturally occurring strains, but apparently no other industry has yet used yeast hybrids commercially. Nor have polyploid yeasts been used commercially, though they have attractive qualities in that they are larger, ferment faster, and give higher yields than diploid yeasts.

The fermentation of various sugars, such as maltose, sucrose, raffinoso and galactose, is under simple genetical control in yeasts, and this has recently been found to extend to the fermentation of maltiotriose, dextrin and starch. Practically any selection of carbohydrate-fermenting abilities could be combined in one yeast, with possible applications both in industry and also for isolation of the individual enzymes.

It is very disconcerting if an industrial yeast changes its properties ; fortunately this is very rare, but brewing yeast does occasionally change its flocculation characteristics. The ability to flocculate is controlled by from one to three pairs of dominant genes which are more than ordinarily subject to mutation. Flocculation generally requires calcium ions, sometimes alcohol, is inhibited by mannose, maltose or glucose, but not by mannitol, raffinose or fructose, is dependent on $p \mathrm{H}$ and on surface tension, and sometimes is achieved only when another mutually flocculent yeast is present. All this is known, but there is much that is not known about the phenomenon and we have not yet got complete control of the balance between too intense and too weak flocculation, either of which may cause technical difficulties. A recent innovation is the deliberate use of two yeast cultures, one flocculent and one dispersed, in a brewery, with control by adjustment of the percentage of each in the mixture used for brewing.

The balance between respiration and fermentation is of importance both to producers of yeast and to industries dependent on the products of fermentation. For the former, intense exponential aeration and exponential feeding give the maximum production of yeast for the amount of carbohydrate used, with no alcohol in the residue. For the latter, aeration is necessary to keep the yeast healthy and vigorous, but too much aeration would lead to loss of alcohol production, so that just the correct amount of aeration is essential.

In a short but lively discussion the comparative values of protein extract from grass and of food yeast were questioned. The flavour of the former was stated to be sickly and unpleasant and the texture of the latter did not compare favourably with roast beef, so that in communities which enjoyed a good mixed diet neither was likely to appeal ; but where dietary protein was low either might form a valuable additive, if not a main course. The function of glycogen in the yeast cell was admitted to be still a mystery, while the aerobic fermentation of glucose by osmophilic yeasts was probably due to a shunt from the main system into one of the many alternative pathways.

\section{R. B. GILliLand}

\section{UNIVERSITY APARTHEID IN SOUTH AFRICA}

$\mathrm{T}$ HE proposals of the South African Government to enforce racial segregation at the universitylevel and to exercise rigid control over projected colleges for non-whites has caused concern and indignation in university circles throughout the Commonwealth and beyond. Some of this concern found expression in a well-attended meeting held at the Caxton Hall in London on November 2 under the sponsorship of the International Committee on Science and Freedom in co-operation with the Association of University Teachers. Dr. J. W. Cook, vice-chancellor of the University of Exeter, presided at the meeting, which by an overwhelming majority passed a resolution expressing admiration and support for those South African universities which are resisting their Government's policy and calling upon the South African Government to heed the cruel and disastrous consequences of its policy and to abstain from its further pursuit. Messages of support for the protest against university apartheid had been received from a number of organizations, including the Senatus of the University of Aberdeen, University of British
Columbia, University of the Philippines, University of the Punjab, University of Delhi, the University College of North Staffordshire, various branches of the Association of University Teachers and the National Union of South African Students.

In his introductory address, Prof. M. Polanyi, chairman of the Committee on Science and Freedom, outlined the Committee's activities during the past three years. He described the apartheid issue as one in a chain of campaigns "always the same in different form". As in other cases, the claim was made that this issue had nothing to do with academic freedom; but if it had, then academic freedom must yield to higher considerations of social interest. Prof. Polanyi suggested that it is an obligation on universities to teach young people those ideas which it is the duty of society to maintain; that any violation of these ideas within the universities cannot be tolerated; and that to demand the participation of universities in a programme of inhumanity, oppression and intellectual dishonesty is a violation of academic freedom. The general philosophy of apartheid, against the 